Estudios Internacionales 174 (2013) - ISSN 0716-0240 • 15-25

Instituto de Estudios Internacionales - Universidad de Chile

\title{
GAS POR MAR
}

\author{
Gas for sea
}

Pascale Bonnefoy*

\section{Resumen}

La actual situación energética en Chile y Bolivia podría convertirse en una oportunidad para que ambos países retomen la fracasada iniciativa de "gas por mar» avanzada hace una década por los presidentes Ricardo Lagos y Hugo Banzer. Desde entonces, el cuadro ha cambiado: la menor demanda de gas desde Argentina y el termino del contrato de exportación de gas a Brasil dentro de algunos años obliga a Bolivia a buscar nuevos mercados y una salida más económica para su gas; Chile, en tanto, entrampado en su dependencia energética, en un costoso sustituto de las importaciones de gas argentino y paralizados dos de sus principales futuros proyectos energéticos necesita fuentes de energía más limpias y menos costosas. Ambos países tienen que despejar un conflicto centenario que mantiene la triple frontera entre Chile, Bolivia y Perú en permanente fricción, en vez de ser un polo productivo trinacional que contribuya a la integración energética de Sudamérica.

Palabras Clave: gas por mar, diplomacia del gas, acceso al mar de Bolivia, relaciones chileno-bolivianas, exportaciones de gas.

Periodista. Egresada del Magíster en Relaciones Internacionales, IEI, Universidad de Chile. pascale.bonnefoy@gmail.com

Recibido el 15 de noviembre de 2013; aceptado el 20 de febrero de 2013. 


\section{Abstract}

The current energy situation in Chile and Bolivia could turn into an opportunity for both countries to resume the failed initiative «gas for sea» advanced by presidents Ricardo Lagos and Hugo Banzer a decade ago. The scenario has changed since then: with the decreasing demand for gas from Argentina and the contract for exporting gas to Brazil due to expire in a few years, Bolivia must seek new markets and a less costly route for its gas exports. Meanwhile, Chile is trapped in its energy dependency, an expensive substitute for its imports of Argentinean gas and two of the country's main future energy projects paralyzed. Chile needs cleaner and cheaper sources of energy. Both countries need to resolve a historical conflict that has maintained the triple border between Chile, Bolivia and Peru in permanent friction, instead of facilitating a tri-national productive pole and the energy integration of South America.

KeYwords: gas for sea, gas diplomacy ,Bolivia access to sea, ChileanBolivian relations, gas exports. 
En 2012 un senador oficialista de Bolivia planteó que se podría reconsiderar la decisión de su país de no venderle gas a Chile, resucitando una fórmula para negociar una salida al mar que llegó a sintetizarse como «gas por mar» y que fracasó estrepitosamente a mediados de la década pasada. Esta vez, sin embargo, las condiciones en ambos países han cambiado y la fuerza de los hechos podría volver a instalar el tema del gas en la mesa de negociaciones con Chile.

«Habría que pensar en exportar (el gas) a otros países, podría ser donde necesitan. Chile podría ser una posibilidad; en Chile hay suficiente mercado y es muy costoso (el gas), pero hay que trabajar de manera integral, porque habría que tocar otros temas, temas bilaterales con Chile, sobre todo el tema marítimo [...] Si nos interesa como país vender gas a Chile a un precio mejor que a Brasil y Argentina, ¿’porqué no?» planteó el jefe de bancada del MAS boliviano, senador Eugenio Rojas. ${ }^{1}$

La proposición fue tildada de «entreguista» por opositores políticos y de «alta traición a la patria» por dirigentes obreros y fue desestimada rápidamente por el gobierno boliviano. Se encendió el discurso anti-chileno pero también sirvió, como era la intención del senador, para abrir un debate que no es

1 Vacaflor, Nancy, «El MAS abre debate sobre el intercambio de 'gas por mar'», Página Siete, 09 marzo 2012, La Paz, http://www.paginasiete.bo/2012-03-09/ Nacional/Destacados/300000109-03-12P720120309VIE.aspx. Consultado 06 septiembre 2012. solo retórico; sino que responde a una realidad inminente que ese país tarde o temprano deberá enfrentar.

Lo que quiso decir Rojas, como explicó después, era que había que ser realista: las circunstancias habían cambiado desde que en 1999 Bolivia y Brasil firmaron un acuerdo para que la petrolera estatal brasileña Petrobras importara gas de Bolivia por un período de 20 años. Hoy esas exportaciones sustentan las arcas fiscales bolivianas, pero tienen sus días contados: tras el descubrimiento de enormes reservas de hidrocarburos dentro de su propio territorio. en el futuro Brasil ya no necesitará tanto gas boliviano. Junto con la reducción prevista de la demanda de Argentina, Bolivia se verá en aprietos por abrir nuevos mercados para su gas y buscar una salida menos costosa para sus exportaciones. Ambos objetivos apuntan a Chile.

Sin embargo, el senador Rojas se encargó rápidamente de desvincular su propuesta de la histórica reivindicación marítima de su país. Rememorando el fracasado proyecto de exportar gas boliviano por un puerto chileno a cambio de un enclave costero sin soberanía, el senador aseguró que había que «separar el tema del gas sin tocar el tema del mar».

«Nosotros no podemos canjear el mar por nuestro gas, eso absolutamente no [...] Eso no nos va permitir ningún ciudadano boliviano [...] Hay que estudiar estrategias si se vende a nuevos mercados el gas, o no, tomando 
en cuenta de que ahora estamos en otras condiciones,» dijo. ${ }^{2}$

Mientras en Chile se guardó silencio, el gobierno boliviano rápidamente desmintió que reflotar la fórmula "gas por mar» fuera un tema en discusión, ya que no formaba parte de la agenda de 13 puntos que desde 2006 sirve de base para negociaciones sobre un conjunto de temas bilaterales. "Tenemos que ser responsables con este tema. Tenemos una agenda de 13 puntos que estamos llevando adelante con Chile y ahí no está este tema,» reaccionó el canciller boliviano David Choquehuanca. ${ }^{3}$

Sería ingenuo pensar que el tema del gas no se ha asomado a las reuniones y conversaciones reservadas o extraoficiales entre las autoridades de ambos países. Sin embargo, en Bolivia incluir el gas como elemento de negociación con Chile sobre un derecho (al mar) que considera intransable sigue siendo un tabú desde que la fórmula discutida

2 Morales, Andrés, «Senador Rojas rechaza interpretación de 'gas por mar' con Chile», Unidad de Comunicación de la Cámara de Senadores de la Asamblea Legislativa Plurinacional de Bolivia, 19 marzo 2012, http://andresmoralese.wordpress. com/2012/03/19/senador-rojas-rechazainterpretacion-de-gas-por-mar-con-chile/. Consultado 06 septiembre 2012.

Agencia ABI, "Choquehuanca: El Tema de Gas por Mar no está en la agenda de 13 puntos», Los Tiempos, Cochabamba, 22 marzo 2012, http://www.lostiempos.com/ diario/actualidad/nacional/20120322/ choquehuanca-el-tema-de-gas-por-marn o - e s t a - e n - l a - a g e n d a de-13_165009_345779.html. Consultado 06 septiembre 2012 . entre los entonces presidentes Ricardo Lagos y Hugo Banzer quedara sepultada en medio del fuerte rechazo popular de la idea de vender gas por un puerto chileno. En efecto un mes antes de que Lagos asumiera la presidencia de Chile en 2000, los gobiernos de ambos países habían comunicado públicamente que las relaciones bilaterales se basarían en una "agenda sin exclusiones», lo que abrió la puerta a que ambos presidentes mantuvieran conversaciones reservadas que derivaron en el diseño de un plan que daría una ruta de acceso a Bolivia al mar, postergando su soberanía sobre ella. Para Lagos fue un arreglo «básicamente comercial» no vinculado con la aspiración marítima de Bolivia (Rodríguez Elizondo, 2006:99).

El plan consistía en que Bolivia exportaría gas a Estados Unidos y México por un periodo de 20 años a través de Pacific LNG, un consorcio internacional compuesto por British Gas (BG), British Petroleum (BP) y Repsol/YPF, empresas que controlaban la explotación de Margarita, la mayor reserva de gas en Bolivia. La primera fase implicaba la construcción de un gasoducto desde Tarija, Bolivia, hasta el puerto chileno de Patillos. Ahí se construiría una planta para producir gas licuado antes de transportarlo por vía marítima a un puerto mexicano, donde se transformaría de nuevo en gas para ser enviado y distribuido en Estados Unidos (Dangl, 2009:157).

En cuanto al dilema político de exportar gas a través de Chile, en circunstancias de que aún no se resolvía 
la reivindicación marítima boliviana, la propuesta incluía otorgar a Bolivia una «zona económica especial» en la II Región, una especie de enclave portuario con plantas de licuefacción y petroquímica y al cual llegaría el gasoducto desde Bolivia. Bolivia no ejercería soberanía sobre esa zona, sino que operaría el complejo bajo un contrato de comodato renovable a largo plazo y sin regalías especiales para Chile. Así, Bolivia gozaría de «las ventajas de la plena posesión sin las responsabilidades de la soberanía» (Rodríguez Elizondo, 2006:102). Además, no requeriría de la aquiescencia de Perú de acuerdo con el Tratado de 1929, como sí lo exigiría la creación de un corredor con soberanía en el norte de Chile.

«La operación gasoducto sería la hebra inicial para tejer, a mediano y largo plazo, redes de confianza rumbo a una normalización total de relaciones. Previendo ese futuro, el analista podría imaginar un poderoso enclave boliviano en Mejillones o Patillos, un restablecimiento paulatino de la confianza mutua, la reposición de las relaciones diplomáticas, un debilitamiento del concepto tradicional de soberanía, cierto entusiasmo peruano por flexibilizar su posición respecto a los territorios que perdiera en la guerra, y, en definitiva, la instalación en la triple frontera de un polo de desarrollo chileno-peruano-boliviano, con vista al mar» (Rodríguez Elizondo, 2006:102).

Cuando este diseño se filtró al público, los bolivianos lo entendieron no como un mero «negocio privado» que privilegiaba los menores costos de exportar a través de Chile en vez de Perú, sino como una renuncia de Bolivia a una salida al mar con soberanía. La mayoría de la población boliviana rechazó el gasoducto a través de Chile, reaccionando con un levantamiento popular que derivó en la renuncia del Presidente Gonzalo Sánchez de Losada ${ }^{4}$ en lo que se conoció como la "guerra del gas». Los sublevados exigían revertir la privatización del sector de hidrocarburos y privilegiar el consumo interno. Una de sus principales consignas se refería a no vender gas a Chile, ni a través de Chile.

Para entonces, las reservas probadas de gas natural en Bolivia se habían decuplicado, gracias a fuertes inversiones en el sector en los años anteriores.

\section{LA DIPLOMACIA DEL GAS}

En este marco asumió el Presidente Carlos Mesa. Políticamente, no le era posible renunciar a la reivindicación de un acceso soberano al Pacífico, so pena de enfrentar un levantamiento similar al que había tumbado a Sánchez de Losada. «La emocionalidad boliviana había reforzado el más furibundo antichilenismo y había que aceptar un antes y un después del 17 de octubre que bloqueaba toda viabilidad al 'non paper' (que no se mencionó explícitamente pero era el referente obvio) que se había

4 Sánchez de Losada había sucedido al presidente interino Jorge Quiroga, quien a su vez había reemplazado a Banzer tras su muerte. 
discutido con Jorge Quiroga (la posibilidad de una zona económica especial, sin soberanía, en Patillos),» explica Mesa (Mesa, 2011).

Mesa repuso la reivindicación irrenunciable de soberanía, utilizando el gas como un instrumento que fortaleciera la posición negociadora de su país. Así las cosas, Chile respondió que si Bolivia buscaba un corredor soberano que pasara por territorio que había sido peruano, tenía que buscar acuerdo con el Tratado de 1929 tenía que buscar el apoyo de Perú. Mesa lo interpretó como una muestra más de la perenne inflexibilidad chilena.

Por primera vez, dice Mesa en un video de su autoría, Bolivia tenía un arma potente para negociar con Chile una salida al Pacífico con soberanía. «No existía ninguna posibilidad de que Chile cambiara su lógica y su actitud per se, por razones morales, por razones de reparación histórica o por razones jurídicas. Solo había un elemento y un argumento que tenía que ver con que por primera vez en la historia, desde que perdimos el mar, Bolivia tenía un arma tangible y real que le permitía negociar con fuerza. Esa arma se llama gas» (Mesa y Espinoza, 2008).

En ese momento era impensable plantear en Bolivia la posibilidad de exportar gas por un puerto chileno sin asegurar previamente una salida soberana al Pacífico. Mesa apostó a las ansias de Chile de contar con ese recurso y lo transformó en una estrategia de negociación. Entretanto, en marzo de 2004 Argentina había comenzado a restringir sus envíos de gas natural a Chile, al verse imposibilitada de abastecer a su propia población. Aunque Chile lo consideró una grave brecha de contratos suscritos con ese país, Argentina resolvió suspender los permisos de exportación de gas natural, aplicando el protocolo adicional al Acuerdo de Complementación Económica suscrito entre ambos países en 1991 sobre el suministro de gas natural. Este protocolo, en vigor desde 1995, condicionaba el otorgamiento de permisos de exportación de gas natural "en la medida que no se comprometa el abastecimiento interno al momento del otorgamiento, si la legislación de las Partes así lo requiere».5

Para Chile la experiencia fue traumática dependía completamente del gas argentino y a partir de los años noventa muchas industrias, vehículos y hogares en Chile habían reconvertido sus sistemas para adaptarse al gas natural, apostando a un suministro abundante y aparentemente ilimitado de energía barata y limpia desde Argentina. Ahora que Argentina le estaba cerrando la llave de paso a Chile, Bolivia vio una oportunidad para aprovechar el aprieto chileno, e involucró a Argentina y a Perú en su favor le ofreció a Argentina compensar lo que le faltaba de gas

5 Artículo 2 del Decimoquinto Protocolo Adicional del Acuerdo de Complementación Económica $\mathrm{N}^{\circ} 16$ entre la República Argentina y la República de Chile sobre Normas que Regulan la Interconexión Gasífera y el Suministro de Gas Natural entre la República de Chile y la República Argentina, Buenos Aires, 07 julio1995. 
para abastecer su mercado interno con sus propias reservas. El acuerdo al que llegaron tenía una salvedad importante: parte alguna de esas exportaciones de gas boliviano podía ser desviada a «un tercer país», léase Chile. No habría ni una molécula de gas boliviano para Chile, explicó Mesa en su momento.

En julio de 2004, Mesa convocó un referéndum sobre la revocación de la Ley de Hidrocarburos promulgada por Sánchez de Losada, el retorno de las reservas de hidrocarburos a control estatal y la refundación de YPFB, recuperando la propiedad estatal de las empresas petroleras capitalizadas. Reforzando su política hacia Chile, Mesa también incluyó la siguiente pregunta: « ¿Está usted de acuerdo con la política del Presidente Carlos Mesa de utilizar el gas como recurso estratégico para el logro de una salida útil y soberana al Océano Pacífico?» El Sí ganó en todos los puntos. ${ }^{6}$

Acto seguido, Mesa involucró al entonces presidente de Perú, Alejandro Toledo, en su diplomacia del gas. En agosto de 2004, ambos firmaron el «Tratado General de Integración, Cooperación Económica y Social para la Construcción de un Mercado Común entre Bolivia y Perú». Desechando el plan Lagos-Banzer, el proyecto bina-

6 Center for Latin American Studies, «Bolivia: Referendum Vinculante sobre la Política Energética del País 2004», Political Database of the Americas, Georgetown University, Washington, D.C., http://pdba. georgetown.edu/Elecdata/Bolivia/ref04. html. Consultado 11 septiembre 2012. cional incluía un gasoducto hacia Perú y la construcción en el puerto de Ilo de una planta de transformación de gas para su posterior exportación a mercados del norte.

«Solo un acuerdo sólido y efectivo entre Perú y Bolivia que cambiara el desarrollo sur peruano y occidental boliviano, con un negocio como el de exportación de gas a México y Estados Unidos, que comprometiera las reservas de ambos países, cambiaría geopolíticamente las cosas con el tercero en discordia de una manera dramática. Si eso se concretaba, nuestra posición frente a Chile variaría 180 grados y permitiría sin dudas un escenario muy diferente con ventaja de Perú y Bolivia sobre Chile, que hasta hoy siempre tuvo la sartén por el mango» (Mesa, 2011).

Sin embargo, el acuerdo fracasó y dio la razón a Chile. "Chile siempre ha partido de un principio hasta ahora correcto: que Perú y Bolivia nunca se van a poner de acuerdo, que nunca van a hacer nada juntos, y que Bolivia, le guste o no le guste, si quiere exportar su gas al Pacífico necesariamente lo tendrá que hacer por un puerto chileno,» afirma Mesa (Mesa y Espinoza, 2008).

Desde entonces, el tema del gas quedó sepultado, al menos oficialmente. Sin embargo, hoy el escenario ha cambiado: Bolivia debe buscar una salida más barata y nuevos mercados para su gas y Chile está entrampado en su dependencia energética, en un costoso sustituto de las importaciones de gas argentino y 
dos de sus principales futuros proyectos energéticos están paralizados.

La situación deriva de hechos imprevistos en 2004, cuando fracasaron las tratativas de «gas por mar». En 2007, Brasil descubrió la existencia de cinco a ocho mil millones de barriles de petróleo y gas natural en la costa, frente a Rio de Janeiro. En mayo de 2012, Repsol anunció que había descubierto un enorme yacimiento de petróleo y gas en Brasil, estimado en más de 700 millones de barriles de crudo ligero y tres trillones de pies cúbicos de gas. Petrobras espera como mínimo duplicar sus actuales reservas en los próximos años, reduciendo su necesidad de importar desde Bolivia.

Por de pronto, Brasil ya está disminuyendo sus importaciones de gas boliviano debido al aumento de la producción interna y la construcción de infraestructura para importar gas natural licuado por vía marítima. Las exportaciones de gas a Brasil son uno de los grandes pilares del PIB boliviano, pero el contrato vence en 2019. De ser renovado, sería en mucho menor escala.

\section{Chile en busca de energía}

Por su parte, Chile enfrenta una dependencia extrema de fuentes extranjeras para su abastecimiento energético. El actual escenario se puede resumir en el siguiente cuadro: una matriz energética sucia -debido a un alto componente de carbón-, que depende en más de $70 \%$ de combus- tible importado, de abastecimiento inseguro y cuyos precios son los más altos de América Latina (Sohr, 2012).

En la perspectiva de la creciente demanda energética esperada para los próximos años, la situación se agravó en 2012: los dos principales proyectos energéticos con que los últimos gobiernos chilenos contaban para satisfacer la demanda eléctrica del país están paralizados. No hay ninguna seguridad de que los dos megaproyectos - la central hidroeléctrica HidroAysén y la termoeléctrica Castilla- se concreten algún día.

Aunque Chile se sacudió de su extrema dependencia de gas desde Argentina, y puede ahora importar gas de distintas partes del mundo, la alternativa ha resultado muy costosa. Luego de que Argentina le redujera el suministro de gas a Chile, a mediados de 2009 Chile comenzó a operar un terminal de recepción, almacenamiento y regasificación de gas natural licuado en la bahía de Quintero (GNL Quintero S.A.) para abastecer de gas natural a la zona central del país. En lugar de depender de la cercana y barata fuente argentina, ahora depende del portafolio de suministro de gas natural de la multinacional británica BG Group, que controla $40 \%$ del proyecto. ${ }^{7} \mathrm{El}$ gas natural que consumen los chilenos llega en barco, traído por BG Group, desde países tan lejanos como

Los otros socios del terminal son ENAP, Endesa Chile y Metrogas, con 20\% de participación cada uno. 
Trinidad y Tobago, Egipto, Nigeria y Guinea Ecuatorial. ${ }^{8}$

En agosto de 2012, GNL Quintero inició la expansión de la capacidad de regasificación del terminal en $50 \%$, con lo que espera, a inicios de 2014, hacer posible la llegada de un significativo volumen de gas natural adicional a la zona central del país. Seguramente es un buen negocio para BG Group, pero no para los chilenos. Aunque el terminal en Quintero sacó a Chile de apuros tras las restricciones de gas argentino, los costos en inversión han sido enormes y el precio del gas para los consumidores se ha disparado, al igual que aquel del resto de las fuentes energéticas de que Chile depende.

La «Estrategia Nacional de Energía 2012-2030» presentada por el gobierno chileno en 2012 menciona muy someramente el gas natural como alternativa de suministro energético a largo plazo. No propone ninguna estrategia para asegurar o explorar un suministro más barato, sino que se limita a comentar las posibilidades del terminal Quintero: «Existe un importante potencial factible aún por desarrollar para nuestros terminales de regasificación de GNL. La disponibilidad del recurso y la expansión de su participación a nivel mundial, junto con las nuevas técnicas y procesos de exploración y producción, muestran al GNL como una alternativa atractiva para las próximas décadas» (Ministerio de Energía de Chile, 2012).

8 Portal de GNL Quintero, http://www. gnlquintero.com/gnl.htm. Consultado 10 septiembre 2012.
No menciona otras posibles fuentes de suministro de gas.

\section{RESOLVER EL «MAGNO \\ PROBLEMA»}

Bolivia necesita nuevos mercados para su gas y requiere urgentemente más inversiones para el desarrollo de su sector de hidrocarburos; Chile necesita una fuente de energía limpia y menos costosa que las actuales. Ambos tienen que despejar un conflicto centenario que mantiene en permanente fricción la triple frontera entre Chile, Bolivia y Perú en vez de facilitar un polo productivo trinacional, y colisiona con los esfuerzos hacia una integración energética en Sud América.

Perú se está adelantando. En julio, el gobierno peruano anunció la licitación para la construcción de un gasoducto desde la planta Malvinas en Camisea, en el centro-norte de Perú, hacia Cuzco. Este gasoducto se ramificará en dos direcciones: una ruta hacia el puerto de Ilo y otra hacia Juliaca, al noreste del lago Titicaca. «La apuesta del gas peruano es abrir mercados en Bolivia y Chile,» afirma al abogado boliviano Saúl Paniagua Flores. ${ }^{10}$

Por su parte, Uruguay también ha ofrecido a Bolivia el uso del puerto de

$9 \quad$ Este mes, la multinacional española Repsol anunció que había descubierto nuevas reservas de hasta dos trillones de pies cúbicos de gas en la zona.

10 Paniagua Flores, Saúl, "Gas Peruano hacia Bolivia», El Deber, 17 agosto 2012, Santa Cruz, 
Montevideo, donde se piensa construir una planta regasificadora, para exportar su gas hacia mercados extranjeros. Reconociendo que no se justificaría invertir en un gasoducto solo para abastecer al pequeño mercado uruguayo, si Bolivia lograra abrir mercados hacia el Atlántico, Uruguay podría servir de "una boca de exportación de gas boliviano", dijo el vicecanciller uruguayo, Roberto Conde. ${ }^{11}$

Más allá de que Bolivia logre abrir mercados en el Atlántico y use puertos uruguayos, la alternativa de Chile como mercado y punto de exportación sigue siendo la menos costosa y la más razonable, aunque más inestable, por consideraciones políticas. A pesar de que el historial de recriminaciones, desconfianzas y ocasional histeria política entre Chile y Bolivia arriesgan la seguridad de cumplimiento de un eventual acuerdo, reponer el tema de la venta de gas a Chile y a través de Chile podría ser una alternativa sensata que además apunte a resolver un pleito centenario.

A diferencia del período de Banzer y Sánchez de Losada, esta vez los hidrocarburos están mayoritariamente en manos del Estado boliviano, lo cual podría facilitar un entendimiento y ami-

http://www.eldeber.com.bo/vernotacolumnistas.php?id=120816224002. Consultado 08 septiembre 2012.

11 Lazcano, Miguel, «Uruguay ofrece un puerto para exportar gas boliviano", $\mathrm{La}$ Razón, 07 septiembre 2012, La Paz, http:// www.la-razon.com/economia/Uruguayofrece-puerto-exportar-boliviano_0_1683431669.html. Consultado 08 septiembre 2012. norar las presiones de privados. Sin embargo, en Chile se presentaría un gran dilema en relación con inversionistas privados extranjeros, al plantear una alternativa claramente más atractiva que el lucrativo negocio que mantiene BG Group en Quintero.

Un eventual acuerdo, que en otros países podría considerarse un arreglo netamente comercial, en el caso de Chile y Bolivia necesariamente debe abordar el grado y modalidad de soberanía boliviana en la ruta de acceso y/o la zona portuaria que se utilizaría para exportar gas. Esto implicaría, en primer lugar, vencer el rechazo visceral del pueblo boliviano a la idea de vender gas a Chile o a través de Chile, flexibilizar el concepto de soberanía en ambos países, fortalecer las medidas de confianza mutua e iniciar una campaña de sensibilización en ambos lados de la frontera. Y ciertamente, requiere que Bolivia abandone su política de «ni una molécula de gas» para Chile.

«El gas ha sido el elemento fundamental de nuestras relaciones bilaterales en este nuevo siglo,» afirma el diplomático e historiador Ramiro Prudencio Lizón, ex cónsul de Bolivia en Arica. "Pero mientras Chile se esmeraba en obtener nuestro gas, Bolivia se encaprichaba en no venderle [...] En vez de esmerarse en llevar el caso marítimo a tribunales internacionales, lo que demorará mucho su solución, el Gobierno nacional debería buscar nuevas negociaciones bilaterales en base al ofrecimiento de gas a Chile; porque debemos tener presente que si este país 
utiliza nuestro gas, estaría muy llano para buscar un arreglo satisfactorio a nuestro magno problema.» ${ }^{12}$

\section{Bibliografía}

Agencia ABI, "Choquehuanca: El Tema de Gas por Mar no está en la agenda de 13 puntos», Los Tiempos, Cochabamba, 22 marzo 2012, http://www. lostiempos.com/diario/actualidad/nacional/20120322/choquehuanca-el-temade-gas-por-mar-no-esta-en-la-agendade-13_165009_345779.html. Consultado el 6 de septiembre de 2012 .

Center for Latin American Studies, «Bolivia: Referendum Vinculante sobre la Política Energética del País 2004», Political Database of the Americas, Georgetown University, Washington, D.C., http://pdba.georgetown. edu/Elecdata/Bolivia/ref04.html. Consultado el 11 de septiembre de 2012.

Dangl, B. (2009), El Precio del Fuego. La Paz: Plural Editores.

Decimoquinto Protocolo Adicional del Acuerdo de Complementación Económica $\mathrm{N}^{\circ} 16$ entre la República Argentina y la República de Chile sobre Normas que Regulan la Interconexión y el Suministro de Gas Natural, Buenos Aires, el 7 de julio de 1995.

Lazcano, Miguel, «Uruguay ofrece un puerto para exportar gas boliviano", $\mathrm{La}$ Razón, 07 septiembre 2012, La Paz, http://www.la-razon.com/economia/ Uruguay-ofrece-puerto-exportar-boliviano_0_1683431669.html. Consultado el 8 de septiembre de 2012.

Lizón, Ramiro Prudencio, «Nuevamente Gas por Mar», La Razón, 28 de marzo de

12 Lizón, Ramiro Prudencio, «Nuevamente Gas por Mar», La Razón, 28 marzo 2012, La Paz, http:/www.la-razon.com/opinion/ colum n is t a s/ Nueva mente-gasmar_0_1585641424.html. Consultado 06 septiembre 2012.
2012, La Paz, http://www.la-razon.com/ opinion/columnistas/Nuevamente-gasmar_0_1585641424.html. Consultado el 6 de septiembre de 2012.

Mesa, Carlos D., «Mi Gobierno y el Mar», Blog de Carlos D. Mesa Gisbert, 18 de abril de 2011, http://carlosdmesa. com/2011/04/08/mi-gobierno-y-el-mar/, Consultado el 7 de septiembre de 2012.

Mesa, Carlos D. y Mario Espinoza (2008). «Bolivia Siglo XX: Una Historia Narrada por Carlos D. Mesa», Plano Medio Producciones, La Paz, http://vimeo. com/22094680. Consultado el 7 de septiembre de 2012.

Ministerio de Energía de Chile, «Estrategia Nacional de Energía 2012-2030», 28 de febrero de 2012, Santiago. http://www. minenergia.cl/estrategia-nacional-deenergia-2012.html. Consultado el 10 de septiembre de 2012.

Paniagua Flores, Saúl, "Gas Peruano hacia Bolivia», El Deber, 17 de agosto de 2012, Santa Cruz, http://www.eldeber.com.bo/vernotacolumnistas.php?id=120816224002. . Consultado el 8 de septiembre de 2012.

Portal de GNL Quintero, http://www.gnlquintero.com/gnl.htm. Consultado el 10 de septiembre de 2012.

Rodríguez Elizondo, J. (2006). Las Crisis Vecinales del Gobierno de Lagos, Santiago: Random House Mondadori.

Sohr, R. (2012), Chile a Ciegas: La Triste Realidad de Nuestro Modelo Energético, Santiago: Random House Mondadori.

Vacaflor, Nancy, «El MAS abre debate sobre el intercambio de 'gas por mar'", Página Siete, 09 marzo 2012, La Paz, http://www.paginasiete.bo/2012-03-09/ Nacional/Destacados/300000109-03-12P720120309VIE.aspx. Consultado el 6 de septiembre de 2012. 